\section{COMPARISON OF PI3K/AKT/MTOR PATHWAY PROFILES AMONGST THREE IMMUNE PHENOTYPES CLASSIFIED BY ARTIFICIAL INTELLIGENCE-POWERED H\&E ANALYZER IN NON-SMALL CELL LUNG CANCER}

${ }^{1}$ Hyung-Gyo Cho*, ${ }^{2}$ Grace Lee, ${ }^{1}$ Hye Sung Kim, ${ }^{3}$ Sanghoon Song, ${ }^{3}$ Kyunghyun Paeng, ${ }^{3}$ Chan-Young Ock, ${ }^{1}$ Young Kwang Chae. ${ }^{1}$ Northwestern University, Feinberg School of Medicine, Chicago, IL, USA; ${ }^{2}$ Northwestern University, Chicago, IL, USA; ${ }^{3}$ Lunit Inc., Seoul, Korea, Republic of

Background The phosphatidylinositol 3-kinase (PI3K)/Akt/ mechanistic target of rapamycin (mTOR) pathway plays a significant role in both tumorigenesis and progression of disease in non-small cell lung cancer (NSCLC). ${ }^{1}$ Increased activation of the pathway, whether in tumor or immune cells, results in an immunosuppressive tumor microenvironment. ${ }^{2}$ Therefore, we looked into how this pathway differs in three distinct NSCLC immune phenotypes.

Methods Lunit SCOPE IO (Lunit, Seoul, Republic of Korea), a deep learning-based hematoxylin and eosin (H\&E) image analytics tool, identifies lymphocytes and quantifies lymphocyte density within the cancer epithelium (CE-Lym), stroma (CSLym), and combined area (C-Lym). We applied Lunit-SCOPE IO to H\&E-stained tissue images of 965 NSCLC samples from The Cancer Genome Atlas (TCGA). Tumors in the lowest tertile of C-Lym were labeled as immune-desert, and the remaining tumors were classified as inflamed and immuneexcluded according to the median of the ratio of CE-Lym to CS-Lym.Utilizing RNA-sequencing data from TCGA, gene set enrichment analysis (GSEA) was conducted to analyze the differences in mTORC1 and PI3K/Akt/mTOR signaling between the subtypes. ${ }^{3}$ We obtained mutational data related to the PI3K/Akt/mTOR pathway from cBioPortal to compare the ratio of functional mutations between the immune phenotypes. $^{4}$

Results The mTORC1 signaling gene set was consistently enriched in immune-excluded, whether compared to inflamed $\left(p_{\text {adj }}<0.01\right.$, normalized enrichment score [NES]: 2.3) or immune-desert ( $p_{a d j}<0.01$, NES: 1.6). However, PI3K/Akt/ mTOR signaling gene set enrichment did not show statistically significant differences between the immune phenotypes. Within the three immune phenotypes, we analyzed three functional mutations: PIK3CA, PTEN, and Akt1 (figure 1). Of the total 112 samples showing the functional mutations of the PI3K/ Akt/mTOR pathway, 53 were immune-excluded, 31 inflamed, and 28 immune-desert. The relation between mutation frequency and the immune subtypes was significant $\left(X^{2}(2)=\right.$ 11.1979, $p<.01)$. The immune-excluded was more likely than the other subtypes to have functional PI3K/Akt/mTOR mutations.

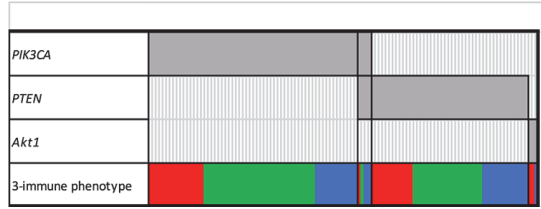

\begin{tabular}{|c|c|c|}
\hline Inflamed & Excluded & Desert \\
\hline $17 / 64$ & $33 / 64$ & $14 / 64$ \\
$(26.6 \%)$ & $(51.6 \%)$ & $(21.9 \%)$ \\
\hline $13 / 49$ & $21 / 49$ & $15 / 49$ \\
$(26.5 \%)$ & $(42.9 \%)$ & $(30.6 \%)$ \\
\hline $2 / 3$ & $0 / 3$ & $1 / 3$ \\
$(66.7 \%)$ & $(0 \%)$ & $(33.3 \%)$ \\
\hline $31 / 112$ & $53 / 112$ & $28 / 112$ \\
$(27.7 \%)$ & $(47.3 \%)$ & $(25.0 \%)$ \\
\hline
\end{tabular}

Abstract 921 Figure 1 The landscape of functional mutation and immune phenotypes regarding PI3K/Akt/mTOR pathway

Conclusions The three tissue phenomic subtypes showed different PI3K/Akt/mTOR pathway profiles, with immuneexcluded having the most mutation samples and the greatest enhancement of mTORC1 signaling gene set. Likewise, tissue H\&E based tumor microenvironment classification by Lunit SCOPE IO can be applied to other hallmark pathways and tumor types, and such further investigation of the tumor microenvironment can provide insights into novel therapeutic avenues.

\section{REFERENCES}

1. Tan AC. Targeting the PI3K/Akt/mTOR pathway in non-small cell lung cancer (NSCLC). Thorac Cancer 2020;11(3):511-8.

2. O'Donnell JS, Massi D, Teng MWL, Mandala M. PI3K-AKT-mTOR inhibition in cancer immunotherapy, redux. Semin Cancer Biol 2018;48:91-103.

3. Liberzon A, Birger C, Thorvaldsdóttir H, Ghandi M, Mesirov JP, Tamayo P. The molecular signatures database hallmark gene set collection. Cell Systems 2015;1 (6):417-25.

4. Cerami E, Gao J, Dogrusoz U, Gross BE, Sumer SO, Aksoy BA, et al. The cBio cancer genomics portal: an open platform for exploring multidimensional cancer genomics data. Cancer Discov 2012;2(5):401-4.

http://dx.doi.org/10.1136/jitc-2021-SITC2021.921 